\title{
The Use of Photographs in Astronomical Instruction
}

\author{
By ${ }^{1}$ M.T.Brück AND ${ }^{2}$ S.P.Tritton \\ ${ }^{1}$ Craigower, Penicuik, EH26 9LA \\ ${ }^{2}$ Royal Observatory Edinburgh EH9 3HJ
}

\section{The Edinburgh Teaching Packages.}

For many years, copies on film of photographs, both direct and through objective prisms, taken with the $1.2 \mathrm{~m}$ United Kingdom Schmidt Telescope, have provided teaching material suitable for universities and colleges (Brück and Tritton, 1988). Table 1 outlines the various types of application to which the photographs may be put. With additional data, some real physics can be injected into the exercises, allowing students to perform quite elaborate projects.

\subsection{Uses for UK Schmidt Telescope Film Copies}

Direct photographs

1. Recognition of objects:

galaxies

minor planets

HII regions, SNRs (in external galaxies)

globular clusters (in the Magellanic Clouds)

2. Statistics

star-counts, for various purposes

number-magnitude counts

star-galaxy counts

galaxies in clusters

3. Changes in position (from more than one photograph)

precession

comet

\subsection{Objective prism photographs}

1. Spectral classification:

coarse classification (of about 100 stars per film)

2. Search for unusual objects:

emission-line stars

carbon stars

planetary nebulae

quasars

A limitation to such purely visual observations is in regard to photometry, where we have to make do with rather rough estimates of magnitude. Measuring the brightnesses or magnitudes of objects is a basic necessity in astronomy, but one that is, ironically, less easy to perform with students than it was ten or twenty years ago. Instruments that were once standard equipment and could be employed on the films - photographic photometers and microphotometers - have fallen into disuse as astronomers receive their data ready processed. For the brighter stars, down to magnitude 13 or 14, magnitudes may be estimated visually to about a fifth a magnitude. This is adequate, however, for our stellar statistics problems (e.g. Fig. 1). 


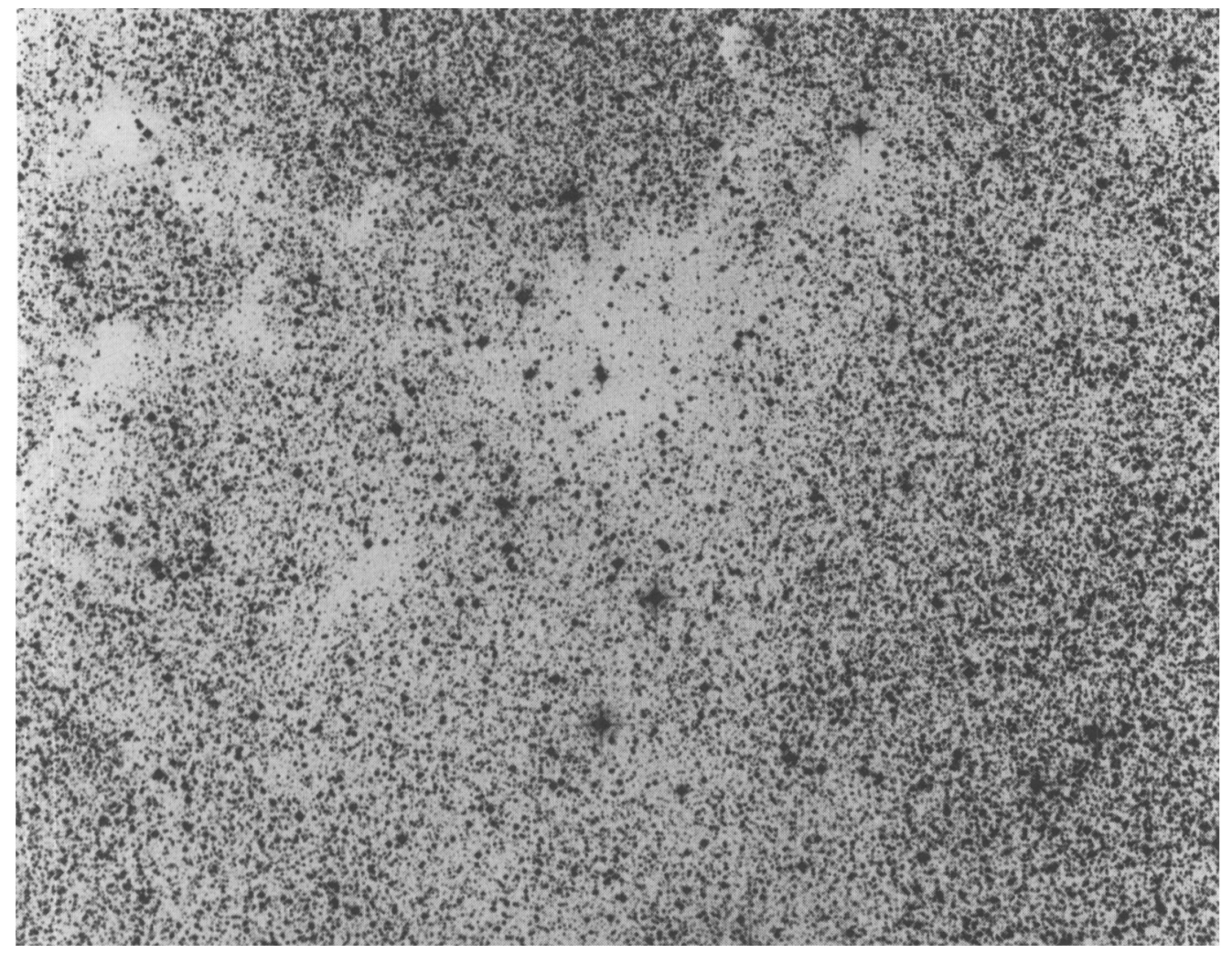

FIGURE 1. A populous region in the galactic plane with an absorption cloud. Star counts yield the extinction in the cloud; photographs of the same region in three different wavebands demonstrate the interstellar extinction law. (C) Royal Observatory Edinburgh.

\section{The use of enlarged reproductions}

Some exercises require the whole field $\left(6.5^{\circ} \times 6.5^{\circ}\right)$ of the films (e.g. Fig. 2). Others in our repertoire do not require a very large area of sky. We have experimented with enlarged photographic prints of smaller areas, incorporated in a book, "Exercises in Practical Astronomy using Photographs"(Brück 1990). Prints, showing all the required detail, were specially produced by the Royal Observatory Edinburgh Photolabs, and excellent half-tone reproductions of these were made by the publishers. Such copies, being enlargements by factors of up to 10 of the originals, mean that linear measurements of images of stars or galaxies can be made with greater ease than from the films.

Some information is bound to be lost in every step of the copying, and in the case of the reproductions in the book, the resolution is limited by the screening dots of $0.2 \mathrm{~mm}$. size. The main loss is in the faintest images, below about 14 , when all images tend to be more or less the same size. The reasons for this are twofold: the loss of information due to the copying process, and the fact that we are not able to make full use of the information within the image, as a proper photometer would. The discrimination between stars and galaxies becomes blurred at the very faintest images, but clusters of not-too-distant galaxies show up well. Objective prism spectra, however, are on the whole not suitable for reproduction on paper prints. 


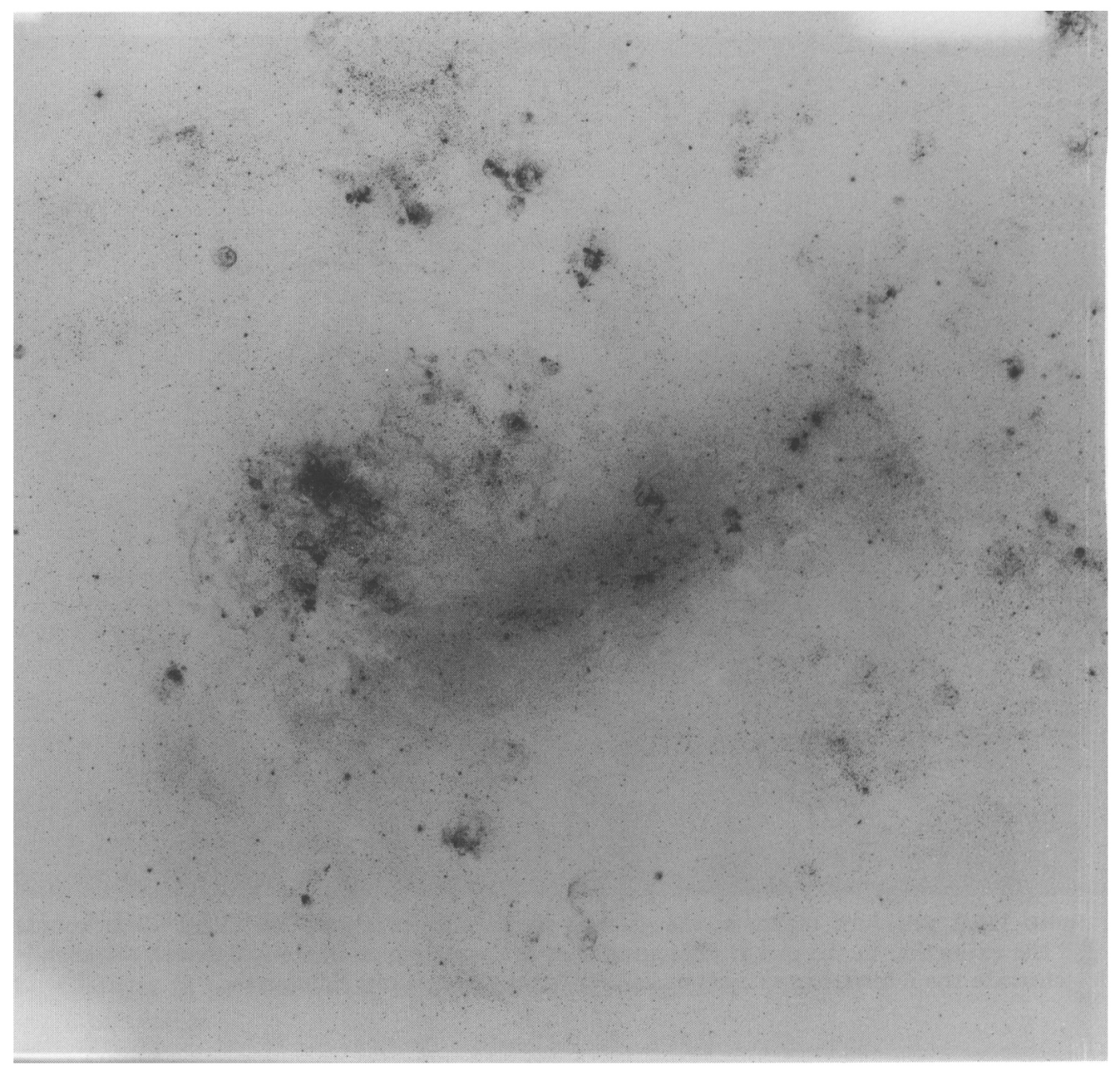

Figure 2. The Large Magellanic Cloud in $U$ (violet) light, filling a full field of the UKST. An objective prism photograph reveals emission lines in many of these features. (C) Royal Observatory Edinburgh

\section{New Trends.}

The title of the conference is "New Trends". As regards our topic, an obvious trend is in the storage of photographs on disk. The entire sky, made up of the Palomar and UK Schmidt telescope surveys, is now available to astronomers on compact disks (The Space Telescope Science Institute 1994). We have done some experiments with this material with a view to its use for teaching purposes. An area of 30 arcminutes square of sky fits conveniently on a 3.5 "computer disk and is readily examined on the screen. Many of the subjects of our exercises are accommodated in such a space. Using only basic graphics supplied with a home computer, we have compared the screen images of stars with those of the photographic enlargements and in the printed reproductions in the book, all derived from the same original photograph.

Figure 3 is an area near the south galactic pole containing a photoelectric sequence of stars with magnitudes ranging from 9.5 (the brightest in the field) to 17.3 (barely visible 


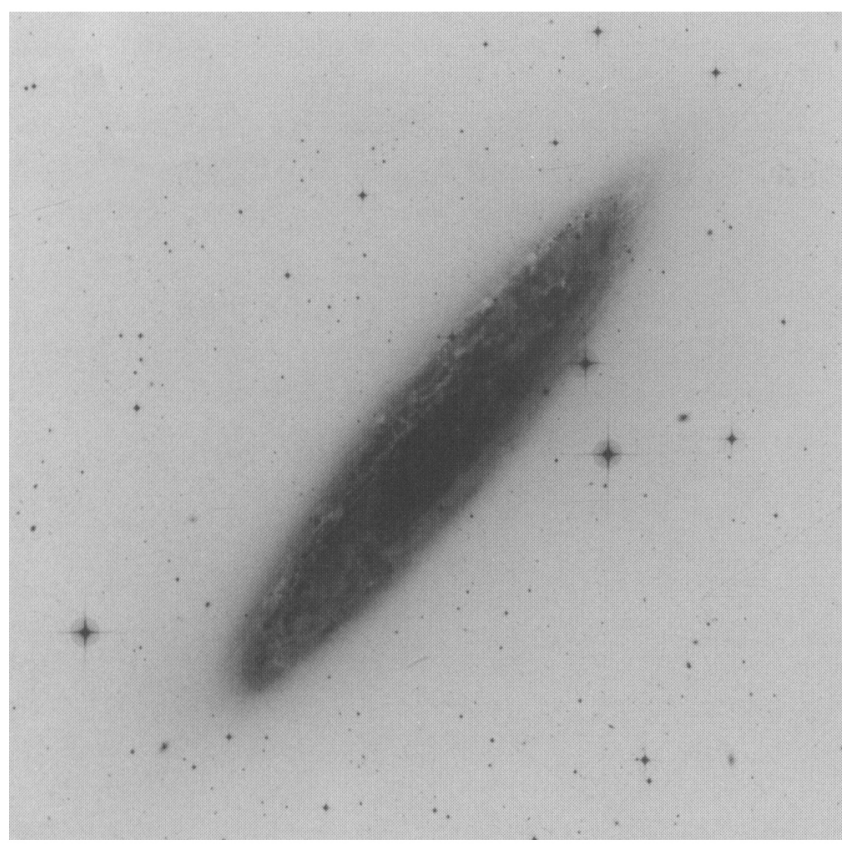

FIGURE 3. A region of half a degree square which contains a photometric sequence ranging from magnitude 9.5, the brightest star, to 17.3 (near the limit of visibility on this photograph). The galaxy is NGC 253. (C) Royal Observatory Edinburgh
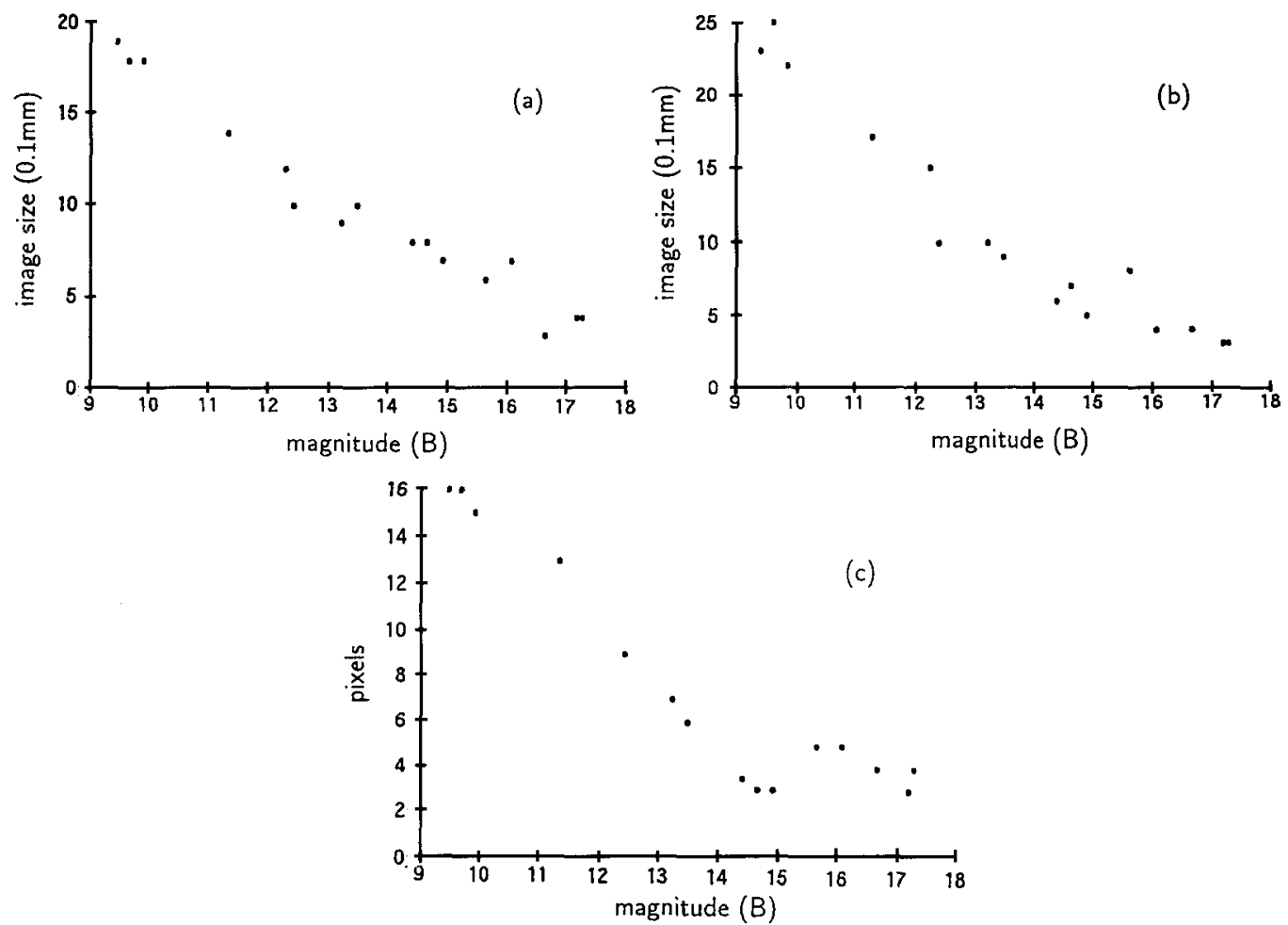

FIGURE 4. Image diameters plotted against magnitude for stars in the field of Figure 3, measured from (a) a photographic enlargement, (b) a printed reproduction and (c) images on the computer screen. 


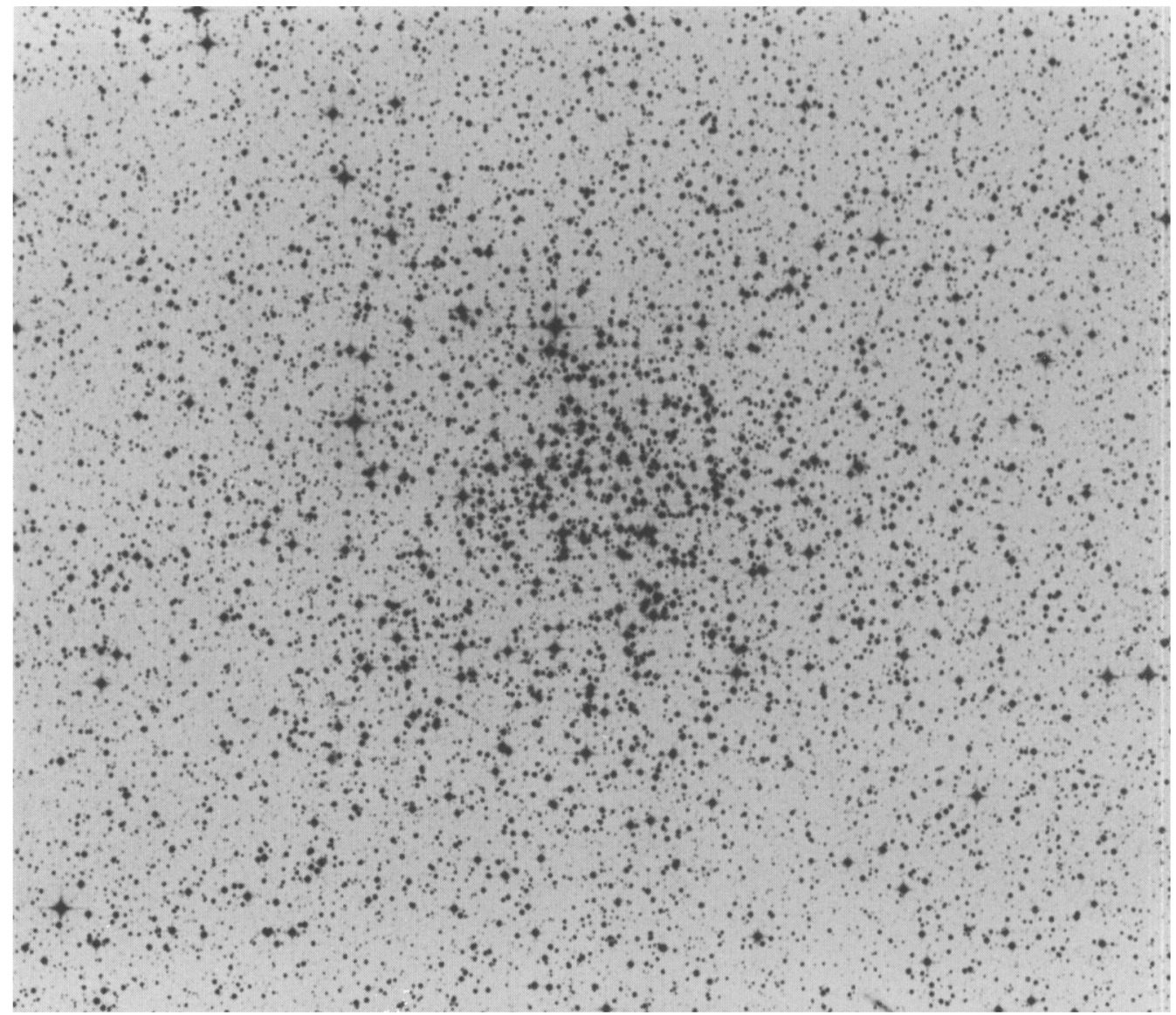

FigURE 5. An open star cluster: an object suitable for recording and analysing on disk. (c) Royal Observatory Edinburgh

on the reproduction). Each star image may be enlarged on the computer screen until it shows the individual pixels, so that image diameters may be read off in pixels. The graphs in Figure 4 show plots of image diameters against magnitude for this sequence using the photographic enlargement (a), the printed version of this enlargement (b) and the image on the computer screen (c). It is seen that the diagrams are all similar and that calibration is usable down to magnitude 13 (or better for the first), but impossible for fainter stars. Though the scatter to this limit is perhaps somewhat larger than we achieve using original films, the statistical exercises are viable in all cases. So too are exercises in measuring galaxy dimensions.

This is very encouraging for the prospect of using disks. Personal computers get cheaper, and software becomes more versatile and more accessible to the ordinary user. There is plenty of useful software commercially available with which to analyse the images on the screen (we have used Autosketch 2.1 for Windows): for example, an area may be divided into small parts, individual images may be enlarged, their dimensions and shapes measured, their numbers noted, and so on.

It is possible to fit a great deal of material on a compact disk. This opens up the prospect of more ambitious exercises than we have done until now, involving, for example, a sample of several clusters of stars (Fig. 5) or galaxies. We are not competing here with 
the experts in the fields of computerised and distance learning but merely showing that useful exercises in astronomy, including some photometry, are feasible on the computer screen. It may well become a favoured method in the future.

Worked examples of some of the exercises may be found in the Proceedings of Colloquium 105 and in "Exercises in Practical Astronomy". Further information on the University and other Packages may be obtained from SBT.

\section{ACKNOWLEDGEMENTS}

Our thanks are due to Photolabs, Royal Observatory Edinburgh, for the illustrations, and to Mr M Read and Dr R Hill for technical advice.

\section{REFERENCES}

Brück M.T. \& Tritton, S.B. 1990, The Teaching of Astronomy, Cambridge, Cambridge University Press, IAU Colloquium no.105, 124-137.

BRücK, M.T., 1990. Exercises in Practical Astronomy using Photographs, Bristol, Adam Hilger IOP Publishing.

BRücK, M.T.Space Telescope Science Institute, 1994,The Digitised Sky Survey, Association of Universities for Research in Astronomy, Inc. USA. 